\title{
空气环境菱铁矿分解氧化过程的穆斯堡 尔效应研究
}

潘永信 朱日祥 平爵云 ${ }^{(4)}$

( 中国科学院地球物理研究所, 北京 100101; (4)北京科技大学材料物理系, 北京 100083)

摘要 在对天然菱铁矿热处理中磁化率变化研究的基础上,利用穆斯堡尔效应分析了空气环 境下菱铁矿的分解氧化过程及其矿物组合变化. 结果表明, 菱铁矿在 $410{ }^{\circ} \mathrm{C}$ 已分解氧化生成 少量磁铁矿, 在 $530{ }^{\circ} \mathrm{C}$ 时完全分解并全部氧化生成磁铁矿, 之后向磁赤铁矿和赤铁矿转化, 赤 铁矿含量随温度的进一步升高而增加。在 $680{ }^{\circ} \mathrm{C}$ 时氧化产物主要由赤铁矿和部分磁赤铁矿组

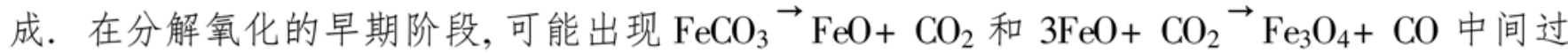
程, $\mathrm{FeO}$ 被立即氧化成磁铁矿而在穆斯堡尔谱中未见. 解释了菱铁矿热处理过程中磁性变化 的原因.

关键词 空气环境 穆斯堡尔效应 分解氧化 菱铁矿

鉴于成岩碳酸盐矿物 $\mathrm{CaCO}_{3}-\mathrm{FeCO}_{3}-\mathrm{MgCO}_{3}$ 三元系的重要理论意义和工业价值, 它们一直 受到岩石学家和矿物学家的重视. 菱铁矿 $\left(\mathrm{FeCO}_{3}\right)$ 是还原环境沉积物中十分常见的矿物之一, 对于未加热菱铁矿人们曾利用差热分析 (DTA)、热磁 $(\mathrm{TM}) 、 \mathrm{X}$ 射线衍射 $(\mathrm{XRD})$ 、傅里叶红外吸 收光谱(FTIR) 和穆斯堡尔谱等多种分析手段进行了研究 ${ }^{[1,2]}$. 菱铁矿的热稳定性较差, 它的 分解过程是研究的热点问题之一, 其热分解动力学 ${ }^{[3]}$ 、在球磨过程中的分解过程 ${ }^{[4]}$ 和氮气环境 的分解作用 ${ }^{[5]}$ 受到关注. Gallagher 等人研究了菱铁矿在真空、氮气和氧气环境的分解作用 ${ }^{\left[{ }^{[}\right]}$. 国内外一些古地磁学家注意到了含菱铁矿岩样的异常磁性质 ${ }^{[7 \sim 9]}$. 我们曾对菱铁矿在空气环 境热处理中的磁学性质变化做了较为详细的研究, 发现磁化率和磁化率各向异性对温度具有 明显的依赖性 ${ }^{[10]}$. 如果古地磁样品中含菱铁矿, 在热退磁处理过程中有可能氧化生成亚铁磁 性矿物, 使样品携带次生热粘滞剩磁, 导致磁性矿物识别和相应古地磁数据解释的错误. 然 而, 目前国内外对菱铁矿在空气环境热处理中磁性与矿物变化对应关系的研究还较少. 本文 在对吉林大栗子菱铁矿样品模拟热退磁过程中磁化率变化研究的基础上, 利用穆斯堡尔效应 分析了空气环境加热菱铁矿的分解氧化过程.

\section{1 实验方法}

实验样品采于吉林大栗子铁矿, 经 X 射线荧光 $(\mathrm{XRF})$ 分析表明, 样品的纯度很高. 样品加 热处理使用 MMTD60 型热退磁炉, 空气环境, 逐步加热温度间隔为 $50 \sim 10{ }^{\circ} \mathrm{C}$, 最高加热温度 $680^{\circ} \mathrm{C}$, 每步在恒温加热 $40 \mathrm{~min}$. 除特别注明外, 文中热处理温度指该样品的最高加热温度. 磁 化率测量使用 KLY-3s 旋转卡帕桥. X 射线荧光分析和磁性测量分别在中国科学院 JICA 矿物 中心和地球物理研究所古地磁实验室完成, 热磁天平测量在国家地震局构造物理实验室完成.

穆斯堡尔效应分析在北京科技大学穆斯堡尔谱实验室完成. 样品先经研磨成细粉, 然后 搅拌在真空油脂中以防氧化. 样品厚度约为每厘米平方有 $10 \mathrm{mg}$ 自然铁. 穆斯堡尔谱仪采用 等加速方式, 实验采取透射几何, 放射源为 $25 \mathrm{mCi}$ 的 ${ }^{57} \mathrm{Co}(\mathrm{Pd})$, 实验温度为室温. 所有测谱均 
用 $\alpha \mathrm{Fe}$ 标准样品作速度标定, 数据经过褶迭处理以提高信噪比并使背景平直. 测量结果用最 小二乘法原理按罗仑兹线型进行拟合. 所得同质异能移位数据均相对于 $\alpha \mathrm{Fe}$ 而言的.

\section{2 结果与讨论}

菱铁矿样品在空气环境加热后的磁化率测量表明 ${ }^{[10]}$, 未加热菱铁矿显示顺磁性, 随着温 度的升高磁化率降低, 而在 $300{ }^{\circ} \mathrm{C}$ 左右开始较缓慢增加, 在 $400 \sim 510{ }^{\circ} \mathrm{C}$ 急剧增加, 510 $530{ }^{\circ} \mathrm{C}$ 出 现最大值, 随后磁化率又较快地降低, 表现出十分明显的温度依赖性. 热磁天平分析揭示出磁 化强度随温度变化具有相似的过程.

选择磁化率发生明显变化的 410, 490, 510, 530, 580, 640 和 $680{ }^{\circ} \mathrm{C}$ 热处理后的样品( 为防止 氧化冷却后用塑料袋密封), 分别做穆斯堡尔效应分析. 穆斯堡尔效应分析的主要结果见表 1 和图 1.

表 1 菱铁矿不同温度热处理产物的穆斯堡尔参数a)

\begin{tabular}{|c|c|c|c|c|c|}
\hline 处理温度/ ${ }^{\circ} \mathrm{C}$ & $\mathrm{IS} / \mathrm{mm} \cdot \mathrm{s}^{-1}$ & $\mathrm{QS} / \mathrm{mm} \cdot \mathrm{s}^{-1}$ & $B_{\mathrm{hf}} / \mathrm{T}$ & 亚谱面积/\% & 主要物相 \\
\hline \multirow[t]{2}{*}{ 未加热 } & 1. $23^{0}$ & $1.79^{6}$ & 0.0 & $100 .^{0}$ & $\mathrm{FeCO}_{3}$ \\
\hline & 1. $22^{8}$ & $1.79^{3}$ & 0.0 & $94 .^{0}$ & $\mathrm{FeCO}_{3}$ \\
\hline \multirow[t]{3}{*}{410} & $0.56^{1}$ & $0.02^{9}$ & $45.1^{4}$ & $0 .^{4}$ & $\mathrm{Fe}_{3} \mathrm{O}_{4}$ (B 位) \\
\hline & $0.44^{2}$ & $-0.12^{1}$ & $49.4^{5}$ & $5 .^{6}$ & $\mathrm{Fe}_{3} \mathrm{O}_{4}$ ( $\mathrm{A}$ 位) \\
\hline & 1. $22^{5}$ & 1. $78^{7}$ & 0.0 & $50 .{ }^{6}$ & $\mathrm{FeCO}_{3}$ \\
\hline \multirow[t]{3}{*}{490} & $0.56^{1}$ & $0.02^{9}$ & $45.1^{5}$ & $11 .{ }^{6}$ & $\mathrm{Fe}_{3} \mathrm{O}_{4}$ (B 位) \\
\hline & $0.32^{3}$ & $-0.01^{0}$ & $49.4^{3}$ & 37.8 & $\mathrm{Fe}_{3} \mathrm{O}_{4}$ ( $\mathrm{A}$ 位) \\
\hline & 1. $23^{1}$ & 1. $78^{8}$ & 0.0 & $28 .^{6}$ & $\mathrm{FeCO}_{3}$ \\
\hline \multirow[t]{2}{*}{510} & $0.57^{0}$ & $-0.03^{2}$ & $45.4^{3}$ & $17 .{ }^{5}$ & $\mathrm{Fe}_{3} \mathrm{O}_{4}$ (B 位) \\
\hline & $0.33^{3}$ & $-0.01^{9}$ & $49.4^{6}$ & 53. ${ }^{9}$ & $\mathrm{Fe}_{3} \mathrm{O}_{4}(\mathrm{~A}$ 位 $)$ \\
\hline \multirow[t]{3}{*}{530} & $0.56^{9}$ & $-0.03^{3}$ & $45.6^{7}$ & $22 .^{3}$ & $\mathrm{Fe}_{3} \mathrm{O}_{4}$ (B 位) \\
\hline & $0.32^{7}$ & $-0.00^{\circ}$ & $49.3^{7}$ & 77. ${ }^{7}$ & $\mathrm{Fe}_{3} \mathrm{O}_{4}$ (A 位) \\
\hline & $0.56^{9}$ & $-0.03^{3}$ & $45.6^{7}$ & $4 .^{1}$ & $\mathrm{Fe}_{3} \mathrm{O}_{4}$ (B 位) \\
\hline \multirow[t]{3}{*}{580} & $0.32^{1}$ & $0.00^{8}$ & $48.8^{0}$ & $29 .^{5}$ & $\mathrm{Fe}_{3} \mathrm{O}_{4}$ (A 位) \\
\hline & $0.38^{5}$ & $-0.08^{9}$ & $50.7^{4}$ & $46 .^{8}$ & $\gamma \mathrm{Fe}_{2} \mathrm{O}_{3}$ \\
\hline & $0.37^{4}$ & $-0.09^{0}$ & $51.6^{1}$ & $19 .^{6}$ & $\mathrm{aFe}_{2} \mathrm{O}_{3}$ \\
\hline \multirow[t]{2}{*}{640} & $0.31^{5}$ & $0.00^{\circ}$ & $48.9^{7}$ & $66 .^{0}$ & $\gamma \mathrm{Fe}_{2} \mathrm{O}_{3}$ \\
\hline & $0.37^{9}$ & $-0.09^{0}$ & $51.2^{2}$ & $34 .^{\circ}$ & $\mathrm{aFe}_{2} \mathrm{O}_{3}$ \\
\hline \multirow[t]{2}{*}{680} & $0.32^{0}$ & $-0.01^{2}$ & $49.4^{7}$ & $22 .^{6}$ & $\gamma \mathrm{Fe}_{2} \mathrm{O}_{3}$ \\
\hline & $0.37^{8}$ & $-0.10^{2}$ & $51.3^{8}$ & $77 .{ }^{4}$ & $\mathrm{aFe}_{2} \mathrm{O}_{3}$ \\
\hline
\end{tabular}

a) 表中: IS 为同质异能移位; QS 为四极分裂; $B_{\mathrm{hf}}$ 为超精细磁场

对于菱铁矿的穆斯堡尔效应来说,最重要的是所有的铁都处在等效晶位上 ${ }^{[1]}$. 图 1 表 明, 未经过热处理的菱铁矿样品, 其穆斯堡尔谱为一双线谱, 同质异能移位为 $1.2 \mathrm{~mm} / \mathrm{s}$, 四极 分裂为 $1.8 \mathrm{~mm} / \mathrm{s}$, 表明菱铁矿为顺磁性, 与 Gil 等人的结果近似. 样品经 $410{ }^{\circ} \mathrm{C}$ 加热处理, 穆斯 堡尔谱的背景上出现六指峰, 表明开始分解出 $\mathrm{Fe}_{3} \mathrm{O}_{4}$, 若各晶位的 $\mathrm{f}$ 因子相同, 则各晶位的亚谱 面积应正比于该晶位的铁原子核数, 由此可计算出分解有 $4 \% \mathrm{Fe}_{3} \mathrm{O}_{4}$. 经 $490{ }^{\circ} \mathrm{C}$ 加热处理, 穆 斯堡尔谱中六指峰的面积明显增加, 而双峰面积明显减少, 并可计算出已分解 $39 \% \mathrm{Fe}_{3} \mathrm{O}_{4}$. 经 $510{ }^{\circ} \mathrm{C}$ 加热处理, 其穆斯堡尔谱中六指峰的面积继续增加, 而双峰面积继续减少, 此时已分解有 $62 \% \mathrm{Fe}_{3} \mathrm{O}_{4}$. 样品经 $530{ }^{\circ} \mathrm{C}$ 加热处理, 其穆斯堡尔谱中双峰消失, 全部为 $\mathrm{Fe}_{3} \mathrm{O}_{4}$, 它具有尖晶石 型晶体结构, 属于立方晶系, 氧离子位于面心立方位置, 阳离子位于四面体位( A 位) 及八面体 位( $\mathrm{B}$ 位). 其中部分 $\mathrm{Fe}^{3+}$ 位于 $\mathrm{A}$ 位, 其余的 $\mathrm{Fe}^{3+}$ 以及 $\mathrm{Fe}^{2+}$ 位于 $\mathrm{B}$ 位. 由于 $\mathrm{B}$ 位的 $\mathrm{Fe}^{3+}$ 及 
$\mathrm{Fe}^{2+}$ 之间存在快速电子交换, 因而谱线重合. 故 只出现 2 套六线谱, 分属于 $\mathrm{A}$ 位及 $\mathrm{B}$ 位, 其超精细 磁场 $B_{\mathrm{hf}}$ 分别为 $49 \mathrm{~T}$ 及 $45 \mathrm{~T}$. 经 $580{ }^{\circ} \mathrm{C}$ 加热处理, 其穆斯堡尔谱出现 4 套六线谱. 表明除 $\mathrm{Fe}_{3} \mathrm{O}_{4}$ 及 $\gamma \mathrm{Fe}_{2} \mathrm{O}_{3}$ 外还有三角晶系结构的 $\mathrm{aFe}_{2} \mathrm{O}_{3} . \quad \gamma \mathrm{Fe}_{2} \mathrm{O}_{3}$ 与 $\mathrm{aFe}_{2} \mathrm{O}_{3}$ 的谱形相同, 但 $\mathrm{aFe}_{2} \mathrm{O}_{3}$ 的超精细磁场比 $\mathrm{\gamma Fe}_{2} \mathrm{O}_{3}$ 的略大, 可以区别. 经 $640{ }^{\circ} \mathrm{C}$ 加热处理后 $\mathrm{Fe}_{3} \mathrm{O}_{4}$ 已消失, $\mathrm{aFe}_{2} \mathrm{O}_{3}$ 量增加. 经 $680{ }^{\circ} \mathrm{C}$ 加热处理 样品的穆斯堡尔谱中 $\mathrm{aFe}_{2} \mathrm{O}_{3}$ 量继续增加, 而 $\mathrm{\gamma Fe}_{2} \mathrm{O}_{3}$ 量继续减少.

Gallagher 等人的研究结果表明 ${ }^{[6,12]}$, 在氧气 环境菱铁矿氧化非常迅速, 只见到赤铁矿; 在真空 环境菱铁矿在 385 和 $410{ }^{\circ} \mathrm{C}$ 分解出金属铁和磁铁 矿, 450 和 $600{ }^{\circ} \mathrm{C}$ 时菱铁矿分解彻底, 以方铁矿和 磁铁矿为主; 在氮气环境生成磁铁矿为主. Seguin 也认为空气中菱铁矿的分解中间产物的氧化速率 远远高于菱铁矿的分解速率, 而总见赤铁矿 ${ }^{[13]}$. Bagin 和 Rybak 及 Loseva 则认为空气中菱铁矿在 $300{ }^{\circ} \mathrm{C}$ 以上会分解形成磁铁矿和磁赤铁矿, 最后转 变为赤铁矿 ${ }^{[14.151}$. 我们的穆斯堡尔谱分析表明, 空气环境菱铁矿分解氧化过程中存在并可见具有 尖晶石结构的磁铁矿和磁赤铁矿中间产物, 它们

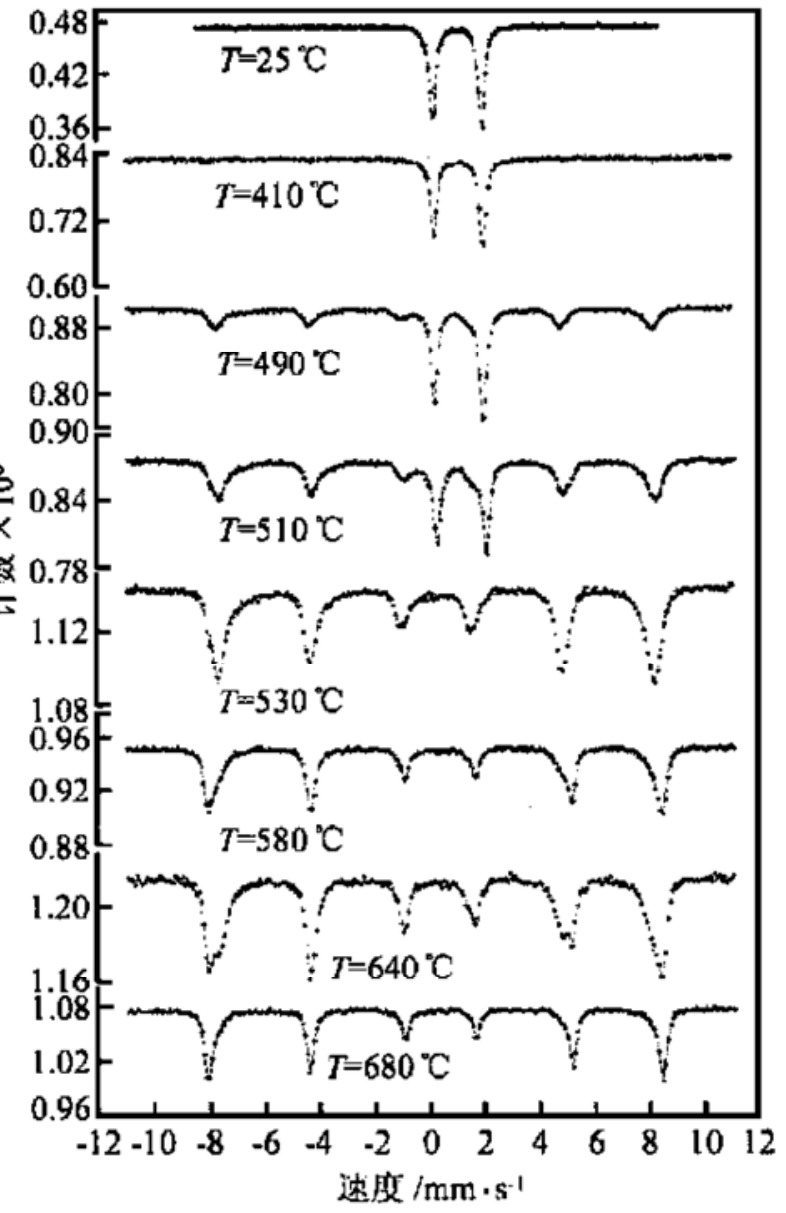

图 1 不同温度热处理后样品的穆斯 堡尔谱(室温) 在一定温度范围具有相对稳定性. 磁铁矿可能由菱铁矿直接分解出来, 也可能通过菱铁矿分 解出的 $\mathrm{FeO}$ 和 $\mathrm{CO}_{2}$ 气体反应(氧化) 生成. 而 $\mathrm{FeO}$ 未见. 岩样 XRD 分析可知, 菱铁矿样品一次 性连续加热至 $720^{\circ} \mathrm{C}$ ( 恒温 $40 \mathrm{~min}$ ), 具尖晶石结构磁铁矿和磁赤铁矿是其主要产物 (图 2). 空 气环境下菱铁矿热磁天平测量结果也显示磁铁矿的存在, $\mathrm{M}(T)$ 曲线显示明显 $580{ }^{\circ} \mathrm{C}$ 的居里温 度.

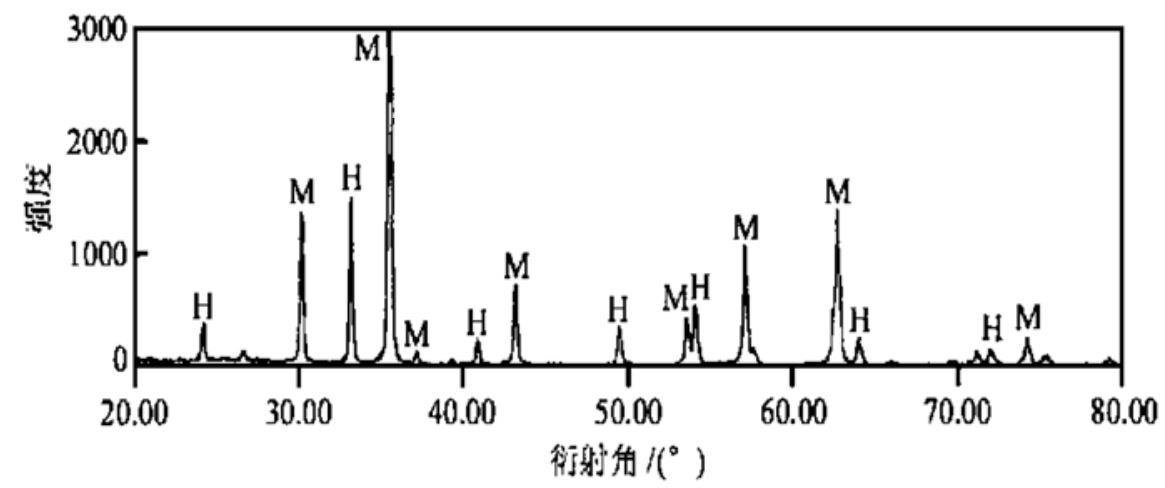

图 2 空气环境菱铁矿样品经 $720^{\circ} \mathrm{C}$ 一次性加热后 (升温速率 $20^{\circ} \mathrm{C} / \mathrm{min}$ ) 的 XRD 谱 图中 $\mathrm{H}$ 代表赤铁矿, M 为尖晶石结构的磁铁矿和磁赤铁矿 


\section{3 结论}

(1) 在空气环境加热过程中, 菱铁矿在 $410{ }^{\circ} \mathrm{C}$ 已分解氧化生成少量磁铁矿, 磁铁矿和磁赤 铁矿为中间产物并具有相对较高的稳定性, 在 $680{ }^{\circ} \mathrm{C}$ 时氧化产物以赤铁矿为主和部分磁赤铁 矿共存, 赤铁矿含量随温度的升高而增加.

(2) 空气环境菱铁矿分解氧化可能由下列过程表示: $3 \mathrm{FeCO}_{3} \rightarrow \mathrm{Fe}_{3} \mathrm{O}_{4}+2 \mathrm{CO}_{2}+\mathrm{CO} ; 4 \mathrm{Fe}_{3} \mathrm{O}_{4}+$ $\mathrm{O}_{2} \rightarrow 3 \mathrm{\gamma} \mathrm{Fe}_{2} \mathrm{O}_{3}+3 \mathrm{aFe}_{2} \mathrm{O}_{3}$, 在分解氧化的早期阶段, 可能出现 $\mathrm{FeCO}_{3} \rightarrow \mathrm{FeO}+\mathrm{CO}_{2}$ 和 $3 \mathrm{FeO}+\mathrm{CO}_{2} \rightarrow$ $\mathrm{Fe}_{3} \mathrm{O}_{4}+\mathrm{CO}$ 中间过程, $\mathrm{FeO}$ 被立即氧化而在穆斯堡尔谱中未见.

(3) 菱铁矿受热分解氧化而转变为强磁性矿物的过程解释了含菱铁矿岩样受热所出现的 磁化率变化. 在海相或湖相沉积物样品的古地磁学研究中, 应特别注意由菱铁矿变化产生热 粘滞化学剩磁的影响. 如果希望通过热处理来分离样品的原生剩磁特征和磁化率各向异性, 则处理温度尽量不要超过 $300{ }^{\circ} \mathrm{C}$.

致谢 本研究工作为国家科委攻关项目和国家杰出青年基金资助项目 (批准号: 49454005), 同时作者感谢国 家地震局构造物理实验室郝锦绮研究员和中科院 JICA 中心刘建明研究员的热情帮助.

\section{参 考 文 献}

1 Nagy D L, Dezsi I, Gonser U, et al. Mossbauer studies of $\mathrm{FeCO}_{3}$ ( siderite). N Jahrbuch Mineralogie Monatshefte, 1975, 3: $101 \sim 114$

2 Gil P P, Pesquera A P, Velasco F. X-ray diffraction, infrared and Mossbauer studies of Fe-rich carbonates. Eur J Mineral, 1992, 4: $521 \sim 526$

3 Zakharov V Y, Adonyi Z. Thermal decomposition kinetics of siderite. Thermochimca Acta, 1986, 102: 101 107

4 Cariado J M, Gonzalez M, Macias M. Influence of grinding on both the stability and thermal decomposition mechanism of siderite. Thermochimca Acta, 1988, 135: 219 223

5 Dubrawski J V. Thermal decomposition of some siderite-magnesite minerals using DSC. Journal of Thermal Analysis, 1991, 37: $1213 \sim 1221$

6 Gallagher P K, Warne S St J. Thermomagnetometry and thermal decomposition of siderite. Thermochimca Acta, 1981, 43: 253 267

7 Ellwood B B, Balsam W, Burkart B. Anomalous magnetic properties in rocks containing the mineral siderite; paleomagnetic implications. J Geophys Res, 1986, 91( 12): 12779 12790

8 周姚秀, 赵西西, 许同春, 等. 含菱铁矿砂岩磁性的初步研究. 地球物理学报, 1991, 34: 343 354

9 刘皇风, 周姚秀, 董金明, 等. 菱铁矿热处理后的异常等温剩磁特征. 北京大学学报, 1994, 30( 1): 61 70

10 潘永信, 朱日祥, 刘青松, 等. 热处理中菱铁矿的磁化率变化与其各向异性交换特征. 科学通报, 1998, 43(12): $1319 \sim 1323$

11 李 哲, 应育浦, 著. 矿物穆斯堡尔谱学. 北京: 科学出版社, 1996

12 Gallagher P K, West K W, Warne S St J. Use of the Mossbauer effect to study the thermal decomposition of siderite. Thermochimca Acta, 1981, 50: 41 47

13 Seguin, M. Instability of $\mathrm{FeCO}_{3}$ in air. Am J Sci, 1966, 264: 562 568

14 Bagin V I, Rybak, R S. The decomposition of siderite by heat Bull Acad Sci USSR. Earth Phys Ser, 1970, 6: 395 398

15 Loseva G V. Crystal structure and magnetic properties during chemica transformations of irorr containing minerals. Bull Acad Sci USSR, Earth Phys Ser, 1972, 10: 688 693 